\title{
A case of inverted follicular keratosis with onset since childhood.
}

\author{
Jianbo Zhong ${ }^{1 \#, ~ W e n b o ~ B u ~}{ }^{2 \#}$, Xu Chen ${ }^{2 *}$, Fang Fang ${ }^{2 *}$ \\ ${ }^{1}$ Department of Dermatology and Venereology, Hangzhou First People's Hospital, Nanjing Medical University, \\ Nanjing, PR China \\ ${ }^{2}$ Jiangsu Key Laboratory of Molecular Biology for Skin Diseases and STIs, Institute of Dermatology, Chinese Academy \\ of Medical Science \& Peking Union Medical College, Nanjing, PR China
}

${ }^{\#}$ These authors contributed equally to this article.

\begin{abstract}
The reports on Inverted Follicular Keratosis (IFK) onset since childhood were rare. Here, we reported 15-y-old male patient with a facial papule occurred since childhood. He was diagnosed as wart and accepted an excision 10 months before this treatment, but the histopathology examination has been ignored. The uniform lesion was recurrent soon. After fusiform excision was performed for the recurred lesion, a histopathology examination showed the feature of IFK. No recurrence has been observed during the follow-up visits for $1 \mathrm{y}$ for this patient after surgical resection. The reports on IFK onset since childhood were rare. This case experience strongly suggests that histopathology examination should not be ignored after the excision of the skin lesion, even though the disease is easy to be acknowledged by clinical diagnosis.
\end{abstract}

Keywords: Inverted follicular keratosis, Surgery, Childhood.

Accepted on March 14, 2018

\section{Introduction}

Here, we reported a 15 -y-old male patient of inverted follicular keratosis on face with onset since childhood. The reports on IFK onset since childhood were rare. This case experience strongly suggests that histopathology examination should not be ignored after the excision of the skin lesion, even though the disease is easy to be acknowledged by clinical diagnosis.

\section{Case Report}

\section{Case}

A 15-y-old male patient came to our hospital for medical treatment because he had suffered from a facial papule for 15 y. When the patient was born, there was a needle-tip-sized papule on the left side of his face. Because he lacked subjective symptoms, the patient did not seek any diagnosis or treatment, and the papule gradually became larger as he grew in age. $\mathrm{He}$ was diagnosed as "wart" and accepted an excision 10 months before this treatment, but the histopathology examination has been ignored. The uniform lesion was recurrent soon. The patient did not suffer from any other diseases, and none of his family members suffered from a similar disease. A dermatological examination found that the papule on the left side of his face $0.6 \times 0.6 \mathrm{~cm}^{2}$ in size; the centre of the papule was ruptured and scabbed with a volcanic crater-like depression, surface telangiectasia, and a hard texture (Figure 1a).
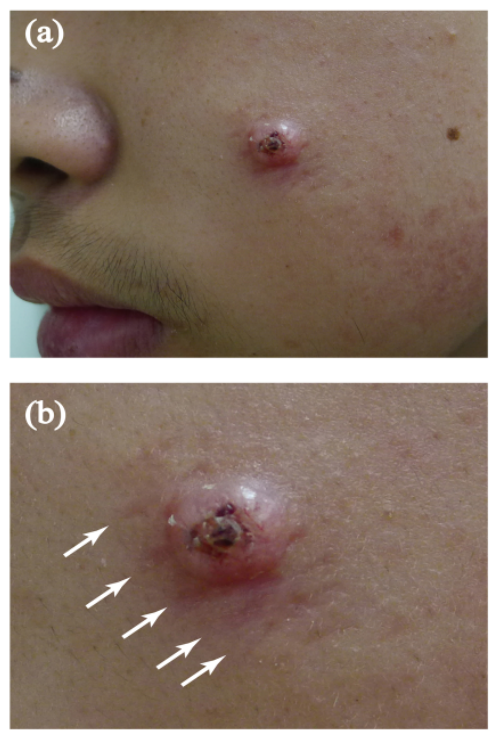

Figure 1. (a) Clinical features before treatment presented a papule on the left side of his face $0.6 \times 0.6 \mathrm{~cm}^{2}$ in size; the centre of the papule was ruptured and scabbed with a volcanic crater-like depression, surface telangiectasia, and a hard texture; (b) The arrows indicated the scars caused by suturation of the first excision. 
After fusiform excision was performed, a histopathological examination showed that the lesion portion grew exogenously and endogenously with distinct borders; the tumour was a mass of epithelial cells with small basaloid cells on the periphery, and squamous fossae were visible in the large squamous cells at the centre (Figure 1b). The disease was diagnosed as Inverted Follicular Keratosis (IFK) (Figure 2).

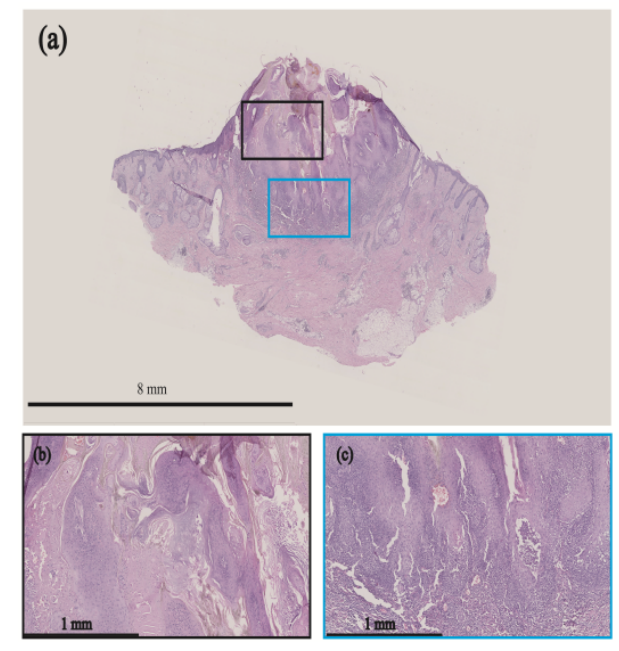

Figure 2. (HE staining 10X) Histopathologic observation manifested that the lesion portion grew exogenously and endogenously with distinct borders; the tumour was a mass of epithelial cells with small basaloid cells on the periphery, and squamous fossae were visible in the large squamous cells at the centre. The observation located in the black frame and blue frame were showed respectively (HE staining 100X). Scale bars were indicated.

\section{Discussion}

The IFK originates from the follicular infundibulum, and the clinical manifestations are isolated papules, nodules, or plaques with a hole-like opening at the centre of the top portion and a keratin-filled interior; these papules are generally observed on the face or neck of elder male patients over $50 \mathrm{y}$ of age and occasionally on the torso or limbs [1]. To our knowledge, the reports on this disease onset since childhood were rare. The aetiology of IFK remains unclear. Some scholars believe that the disease is a type of independent tumour, an old verruca vulgaris, or a stimulated form of seborrheic keratosis that grows endogenously [2-4]. Mehergan had reported on 100 IFK cases in which 68 were male patients, 32 were female patients, and the youngest age of onset was $11 \mathrm{y}$ [2] (Supplementary Figure 1).

Although the clinical manifestations of IFK have certain features, there is a lack of specificity. Diagnosis is mainly based on the histopathological examination results. This case experience strongly suggests that histopathology examination should not be ignored after the excision of the skin lesion, even though the disease is easy to be acknowledged by clinical diagnosis. In addition, because of the age of onset featured in the patient in this case, attention should be paid during diagnosis to differentiate IFK from the following diseases: (i) Verruca vulgaris: characterized by the presence of vacuolization and parakeratosis in the cells of the upper stratum granulosum and the stratum spinosum; (ii) Keratoacanthoma: characterized by dominative exogenous growth, lesions almost completely comprised of squamous cells, and the presence of atypical cells and mitotic figure. Because childhood-onset of keratoacanthoma has been reported, attention to differentiation is necessary during diagnosis [5].

\section{Acknowledgment}

Wenbo $\mathrm{Bu}$ and $\mathrm{Xu}$ Chen are supported by CAMS Innovation Fund for Medical Sciences (CIFMS-2017-12M-1-017) and the PUMC Youth Fund and Fundamental Research Funds for the Central Universities (3332015116, 3332014008, 2016RC320005, 2016ZX320014)

\section{Conflict of Interest}

The authors have no conflict of interest to declare.

\section{References}

1. Armengot-Carbo M, Abrego A, Gonzalez T. Inverted follicular keratosis: dermoscopic and reflectance confocal microscopic features. Dermatology 2013; 227: 62-66.

2. Mehregan AH. Inverted follicular keratosis is a distinct follicular tumor. Am J Dermatopathol 1983; 5: 467-470.

3. Spielvogel RL, Austin C, Ackerman AB. Inverted follicular keratosis is not a specific keratosis but a verruca vulgaris (or seborrheic keratosis) with squamous eddies. Am J Dermatopathol 1983; 5: 427-442.

4. Lever WF. Inverted follicular keratosis is an irritated seborrheic keratosis. Am J Dermatopathol 1983; 5: 474.

5. Kumar V, Kumar H, Thappa DM. Multiple keratoacanthoma with neonatal onset in a girl. Pediatric Dermatol 1999; 16: 411-412.

\section{*Correspondence to}

$\mathrm{Xu}$ Chen

Jiangsu Key Laboratory of Molecular Biology for Skin Diseases and STIs

Institute of Dermatology

Chinese Academy of Medical Science \& Peking Union Medical College

PR China

Fang Fang

Jiangsu Key Laboratory of Molecular Biology for Skin Diseases and STIs

Institute of Dermatology

Chinese Academy of Medical Science \& Peking Union Medical College

PR China 